\title{
Augmented Reality Advertising: An Impactful Platform for New Age Consumer Engagement
}

\author{
Poonam Singh ${ }^{1}$, Mrinalini Pandey ${ }^{2}$ \\ ${ }^{I}$ Department of Management Studies, Indian School of Mines, Dhanbad, India \\ ${ }^{2}$ Department of Management Studies, Indian School of Mines, Dhanbad, India
}

\begin{abstract}
Augmented Reality is the latest buzz in the advertising arena. In augmented Reality, advertisers have found a unique and interactive way to engage audience with the brand. On the other hand it keeps users in charge of the conversation process, letting them retrieve the information they need, when they need it and wherever they need it.

This paper aims at studying why Augmented Reality makes a good choice for the marketers to be used in their advertising campaigns by reviewing the available literature and finding out customers' preferences for media consumption by means of primary data collection. Based on the findings authors suggest reasons why Augmented Reality advertising is one of the best options available to circumvent the clutter in the traditional media.
\end{abstract}

Keywords: Advertising, Augmented Reality, consumer engagement, media-habits, multi-screen audience

\section{INTRODUCTION}

Electronic media consumption is not restricted only to Television screens today. We now have a multiscreen environment. Audiences have variety of options available in the form of computers, smartphones, tablets etc. This has led to fragmentation, and old ways of advertising are rendered ineffective. This does not mean that television has become obsolete. Not so at least in a country like India where infrastructure support is low and we are not yet a 'digital first' market. Nonetheless the uprising of the digital as a medium for advertising cannot be ignored. It may be attributed to the increase in internet users in the country (TRAI pegged the number of internet subscribers at 164.81 million as of March 31, 2013 with seven out of eight accessing it from their mobile phones) and to the increase in literacy levels. Currently majority of consumers' interaction with the media begins with one of the screen options and the rest is through the radio, print or outdoor. Another interesting trend that is observed is that of overlapping consumption [1]. According to researches consumers are squeezing in 12 hours of media exposure in say about 9 hours. They are watching lesser TV than in the past and are spending more time looking at other screens and at times simultaneously. For instance, playing with their smartphones while watching television or if an advertisement in the television arouses their interest, they immediately surf about it on the Internet, mostly accessed through their smartphones. This has some serious learning and implications for the marketers. It makes sense for an intelligent advertiser to be present on the web before the customer reaches there via another media say print or electronic. Multiple digital touch points (four screens viz television, personal computer, tablets and mobile phones) are available today to the consumer over which the online shopping experience can be spread.

Also previous studies have indicated that only if a customer is truly engaged with a brand, will he have a positive feeling about it and the association may result into a sale. Marketers are finding it difficult to get attention of their audience and retain it with much digital noise around [2]. In this context, Advertising industry is rightly excited about Augmented Reality advertising because it has both the qualities embedded in it. One it is an interactive and engaging platform and it is available on the users' mobile phone which has become an extension of one's personality these days, especially for the younger generation.

This paper studies the changes in the media consumption and its implications for the marketers and goes on to suggest why Augmented Reality could be an answer to the problem of engaging customers meaningfully.

\section{REVIEW OF LITERATURE}

Technological transformation and automation of delivery has brought about a revolution in the advertising industry [3]. The digital era brought near constant change for the marketers. Fragmentation caused many channels to erode while others saw an increase in audience as stated in the Econsultancy report, 2010. It is becoming increasingly important for marketers to stay connected with their audience visiting multiple screens, particularly to reach out the evening crowd who seldom watches television without a smartphone or a tablet within his reach [4]. The consumer is engaging with content across a variety of devices simultaneously and hence posing a challenge for the marketer. 
Mobile advertising seems to be an industry that's beginning to explode especially in India where millions of mobile subscribers are getting added every month. Mobile phone is a classic mix of great choice and convenience in media consumption with people hardly leaving home without it (CellStrat, 2008). Today, mobile devices sense your environment laced with GPS, camera, Bluetooth, wi-fi, microphones, light-sensors, etc. diminishing the boundary between the physical and digital worlds [5].

With the advent of smart phones, mobile has become a platform for one of the most interesting technologies of today - Augmented Reality. Augmented Reality is continuing what was started by the Internet and all is about providing experiences [6]. Obst and Troller (2009) [7] have said that "by superimposing virtual imagery, sound and theoretically even other sensorial enrichments over real-world environments in real-time, augmented reality serves as a tool to ever more enhance a human being's awareness and performance". Biocca , et.al, 2007, [8] suggest that knowledge of objects or situations of the environment as provided by Augmented Reality could be very critical for the user. Mobile Augmented Reality new opportunities based around hyper local marketing because consumers are moving away from traditional media and most purchase decisions are driven by social media and they are reluctant to pay for digital content and want advertising funded media [9].

\section{HYPOTHESIS}

To test whether Augmented Reality has any impact on the consumers today with respect to the changing media consumption patterns and dynamic marketing scenario following hypothesis was formulated: Ho: Actual use of Augmented Reality advertisement applications is independent of the kind of advertisement preferred.

\section{METHODOLOGY}

This paper aims at studying the media consumption patterns of customers today and suggesting how Augmented Reality advertising can help marketers to reach out to the multi-screen audience and engage them. Available literature was reviewed for the purpose and valuable insights were gained about the media consumption patterns of today's consumer. The authors tried to authenticate the same by conducting a consumer survey and collecting primary data. A questionnaire was prepared and data was collected by posting the questionnaire online on Google Docs and e-mailing the link to the sample. The sample consisted of people in various age groups ranging from 18 years to 60 years and above. They came from different income groups and professional backgrounds. Convenience sampling was used and a total of 94 sample filled the questionnaire. They answered questions regarding their media habits such as time spent with different media (television, radio, newspaper, Internet, mobile, social media etc.). They were also asked questions regarding the kind of advertisements preferred by them and the media that made most impact on them. The questionnaire also enquired whether the respondent has experienced Augmented Reality Advertising ever or not and if he hasn't, whether he intends to download such an application ever in the future. The responses thus collected were compiled to arrive at the results.

\section{AUGMENTED REALITY AND ITS POTENTIAL FOR ADVERTISING}

Augmented Reality technology has been around for quite some time now. However advertisers are beginning to take notice of the technology recently and we see more and more advertising campaigns using the technology. Augmented Reality refers to augmenting a real world environment with computer generated digital data in the form of images, sound, 3D model etc. These applications can be downloaded with devices such as laptops with web-cam, tablets and mobile phones with camera with a working Internet connection. Usually people use their smartphones to download them. To experience the advertisement, the user has to download an application from the advertiser's website and point the mobile phone camera to a marker or image (that is programmed to trigger the application) and can see a whole new world on the screen.

The potential for Augmented Reality advertising lies within its ability to engage the audience and reach them in a way that other mediums cannot [10]. The very fact that the message is not forced on to the user and he downloads it at his own will, begins the engagement with the brand. Also the user is completely in charge of the message, navigating it to his liking. Thus seeking only the information that he wants. Moreover it enables the marketers to send very targeted messages as these messages are delivered to people who access the messages themselves. One can send geographically specific ads using the technology that promote the nearby businesses to the people.

Google Glass, the latest addition to the field of Augmented Reality has managed to generate a lot of excitement and buzz around the industry. Such innovations can possibly increase the efficacy of advertising. Without doing anything physically, audience would receive targeted promotional messages from the advertisers by merely wearing these glasses. 


\section{FINDINGS AND DISCUSSIONS}

The consumer survey threw light on the media consumption pattern of the consumers. The respondents were asked about the time spent on each of the different media by them in a typical day. The authors found out that amongst different media options available, respondents spent the least time listening to radio with 59\% saying that they listen to radio for less than an hour and 39\% indicating that they listen to radio between one to three hours. Television is still a popular medium with a consumption of up to 3 hours daily by $61 \%$ respondents and about $3-5$ hrs by $21 \%$ of the respondents. Of the total respondents, $80 \%$ of the respondents spent between 1-3 hrs with the newspaper. Browsing the Internet topped the list with almost $90 \%$ of the respondents agreeing to having spent some time on it daily. $47 \%$ respondents spent up to 3 hours on the net, $32 \%$ spent up to 5 hours and a decent $20 \%$ spent more than 5 hours browsing the Internet. $56 \%$ respondents said that they spent up to 3 hours playing / surfing on their mobile phones where as $19 \%$ used it for up to 5 hours. $66 \%$ respondents spent up to 3 hours on the social media and $17 \%$ spent up to 5 hours on it. The data suggests that though television viewing is still very popular across age groups, internet is catching up fast. People are spending considerable amount of time online whether on web or through their mobile phones (Fig. 1).

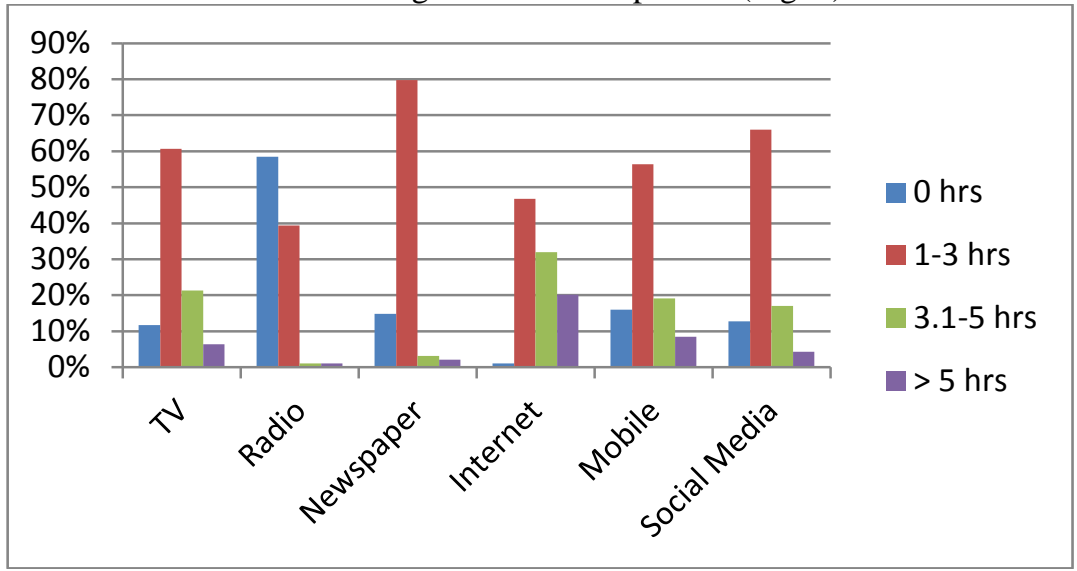

Fig. 1: Media consumption pattern

When asked as to what kind of advertisements appeal more to them, $50 \%$ of the respondents said that moving, action-oriented and interactive advertisements are preferred by them followed by words with audio and visual as suggested by 35\% respondents (Fig. 2). This suggests that consumers no longer want a passive monologue for an advertisement; instead they are looking for some kind of interactive format, which engages them with the brand, gives them information and may be allows them to give their feedback or suggestions simultaneously.

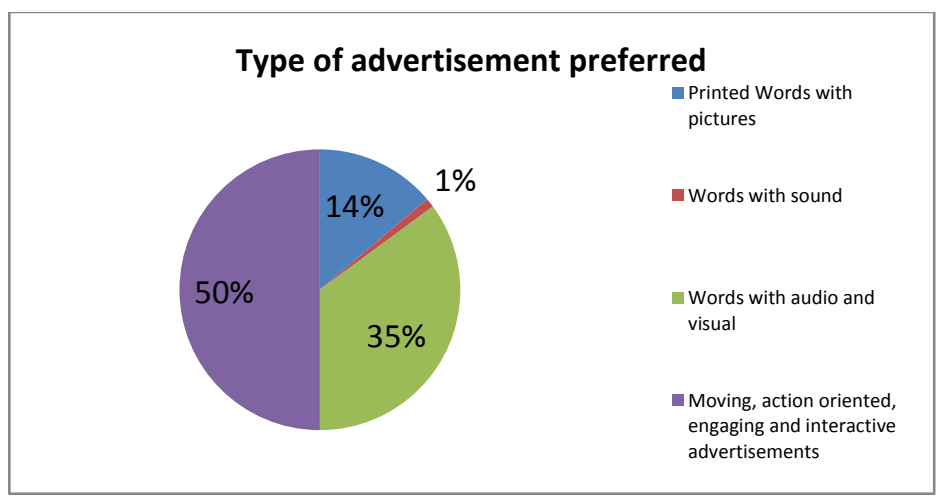

Fig. 2: Type of advertisement preferred

Television as an advertising medium still has maximum impact with 52\% respondents opting for it followed by $21 \%$ saying that they are influenced by word of mouth and $16 \%$ opting for Internet.

When asked what made for a good advertisement according to them, 33\% opted for a good storyline/concept followed by adequate information about the product (26\%). Customer wants all the necessary information about the product without compromising on the creativity component. 


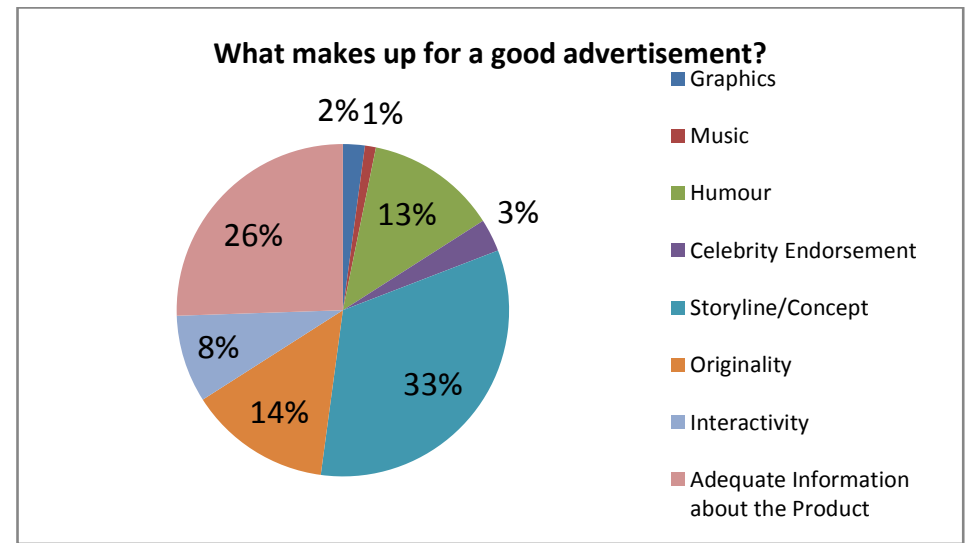

Fig. 3: What makes up for a good advertisement?

Out of the total respondents, an encouraging $46 \%$ have experienced Augmented Reality advertisements at least once and $90 \%$ of these have found these advertisements engaging. $65 \%$ of the non-users indicated that they would like to download such applications in the future suggesting that though the current awareness levels about the technology use in advertising is low, there is enough curiosity around the medium amongst the consumers and if promoted properly can fetch good results for the advertisers.

To test the hypothesis, the response of the respondents about the kind of advertisement preferred was cross tabbed with whether they have downloaded any Augmented Reality advertisement application or not and Chi-square test was performed.

Table 1: Result of Chi-square test of preference of advertisement types and the actual use of AR advertisement

\begin{tabular}{|l|c|c|}
\hline & $\chi^{2 \mathrm{cal}}$ & $\chi^{2 \mathrm{tab}}$ \\
\hline Kind of advertisement preferred & 19.61 & 14.067 \\
\hline
\end{tabular}

At $5 \%$ significance level

From the above results it is clear that at $5 \%$ level of significance $\chi 2 \mathrm{cal}>\chi 2$ tab.

Hence the null hypothesis is rejected. In other words, there does exist a relationship between the kind of advertisement preferred and actual usage of Augmented Reality advertisement applications. The consumer survey data also suggests that majority of the respondents who have indicated a preference to the actionoriented, engaging and interactive advertisements have downloaded Augmented Reality applications. This indicates that Augmented Reality satisfies the consumers need for engaging and interacting with the brand in the manner that they want to.

\section{CONCLUSION}

The findings from the literature review and the consumer survey suggest that though the traditional media still enjoys maximum patronage and is suitable for Indian markets; audience is spending considerable amount of time online, whether on computer or mobile. Also consumers no longer prefer passive monologues by the marketers but want to be a part of the conversation with the brand. They want to experience more interactive, engaging kind of advertisements which provide them with all the information necessary to make a purchase decision. As suggested by the literature, marketers now have a multi-screen challenge before them. Audience is simultaneously watching and seeking information from a variety of screens. Also the mobile phone has become an inseparable part of the personality of consumers. It is therefore a challenge for the advertiser to choose the right medium to reach the consumer. It would make sense if they are able to integrate various media in a way such that they do not miss out on the reach offered by the traditional media and yet take advantage of customers' new found interest in the interactive, digital medium which is mobile.

Augmented Reality has the potential of becoming an answer to tackle this problem. It is an interactive medium which keeps the users in control of the experience enabling them to retrieve only the information that they want. They can access the advertisement from their mobile phones from wherever they are using the technology. The markers can be made available either in the print medium or on 'screens' or enabling a physical object such as the product pack to trigger the application. The traditional media especially television combined with the social networking platform can be used to create a buzz around the campaign. This will integrate the two while empowering the customer.

Augmented Reality has many advantages that make it an attractive proposition for the marketers. To start with, it is a unique concept, relatively new in the market and has been able to generate enough curiosity around itself. It is possible for the advertiser to personalise the communication as per the user since user is in 
charge of the navigation. There is no restriction of space or time like the traditional media and the advertiser can fit in more content hence providing sufficient information to the user. If done properly, An Augmented Reality advertisement has the potential of going viral [11]. One satisfied consumer would recommend it to his friends thus increasing number of scans. It gives a richer experience to the user by increasing interaction and engagement.

It not only makes a brand more innovative and responsive but also increases its perceived value [12]. Augmented Reality is available on the mobile and thus becomes very personal, given that the users of smartphones are increasing every day. It appeals especially the younger tech-lovers who are usually sceptical of traditional advertising.

An Augmented Reality application can be developed at a much lower cost than those on other traditional media. Also the advertisers can measure the response to the advertisement in real time. There are analytics available for the purpose which let the advertisers find out how many people have scanned the advertisement, from where and using which handset. Also in some cases the demographic details of the person can also be accessed. Thus enabling the advertisers to understand their customers better.

Whether it is an automobile advertisement that the consumer has downloaded to test drive a new model or trying a dress virtually that she wishes to purchase, or playing a game, Augmented Reality campaigns stays with the customer in a way that traditional media fails to deliver. The advertisement no longer remains a commercial but becomes a kind of game. Still it manages to convey the message intended by the advertiser. Isn't it the whole idea of advertising?

\section{REFERENCES}

[1] Zigmond, D and Stipp, H, Multi-taskers may be advertisers best audience, Harvard Business Review, January-February, 2011, 1-3

[2] Thibeault, J, Engaged Audience: reaching your audience across multiple screens, retrieved from http://www.tvtechnology.com/article/engaged-audience-reaching-your-audience-across-multiple-screens/222210, 2013, retrieved on November 18, 2013

[3] Goldfarb, A and Tucker, C, How do advertising standards affect online advertising?, http://ssrn.com/abstract=1745645, 2011, 1-38

[4] Hines, M, The multi-screen consumer, why connecting to your audience is crucial, retrieved from http://blog.gannettlocal.com/blog/bid/314535/The-Multi-Screen-Consumer-Why-Connecting-to-Your-Audience-is-Crucial, 2013, retrieved on November 18, 2013

[5] Tirri, H, Future mobility building the path forward, retrieved from https://research.nokia.com/people/henry_tirri, 2008, retrieved on October 4, 2012

[6] Perey, C, Augmented Reality. Continuing what the internet started, retrieved from http://www.perey.com/ARDevCampZurichMarch1/Overview-of-Augmented-Reality-March1.pdf, retrieved on February 10, 2011

[7] Obst, B and Troller, L, Augmented Reality, retrieved from http://wiki.informatik.huberlin.de/nomads/images/9/96/Augmented_Reality.pdf, 2009, retrieved on November 11, 2012

[8] Biocca, F et.al, Attention issues in spatial information systems: directing mobile users' visual attention using augmented reality, Journal of Management Information Systems, 2007, 23(4), 163-184

[9] Damani, C, Hyperlocal marketing for mobile augmented reality, retrieved from http://www.perey.com/MobileARSummit/AcrossAirHyperlocal-marketing-for-MobileAR.pdf, 2010, retrieved on March 11, 2012

[10] Leach, D, Augmented reality and its potential for advertising, retrieved from http://blog.ad-tech.com/augmented-reality-and-itspotential-for-advertising/, 2013, retrieved on November 17, 2013

[11] Smith, A, 5 benefits of augmented reality marketing, retrieved from http://socialmediatoday.com/alex-smith/242184/5-benefitsaugmented-reality-marketing, 2010, retrieved on November 21, 2013

[12] Andy, Unlocking the potential of augmented reality, retrieved from http://www.enginecreative.co.uk/insights/unlocking-potentialaugmented-reality, 2012, retrieved on September 21, 2013 